\title{
Equilíbrio mecânico de uma barra: uma proposta para o laboratório de física
}

\section{Mechanical equilibrium of a rod: proposal for the physics laboratory}

\author{
Ubaldo Martins das Neves, Thiago de Oliveira Calsolari ${ }^{\dagger}$
}

Universidade Federal de São Carlos, Campus Lagoa do Sino, Rodovia Lauri Simões de Barros, Km 12-SP-189, Bairro Araçu-Buri-SP

\begin{abstract}
Resumo
Descrevemos aqui um experimento didático e de baixo custo visando a aprendizagem dos conceitos físicos de resultante de forças e torques, envolvidos no estudo do equilíbrio de um corpo extenso. Para tanto, uma régua de plástico é pivotada em uma de suas extremidades e submetida a uma carga na outra extremidade. O sistema é mantido em equilíbrio horizontal pela ação de uma terceira força direcionada para cima, exercida por um dinamômetro. Cálculos da intensidade da força exercida pelo dinamômetro em função da carga suportada pelo sistema e em função do ponto de aplicação do mesmo na barra são comparados com os dados experimentais revelando perfeita concordância.
\end{abstract}

Palavras-chave: Condições de equilíbrio para um corpo rígido. Torque e força. Ensino de física.

*ubaldo@ufscar.br

†thiagocalsolari@yahoo.com.br 


\begin{abstract}
This article describes a low coast and didactic experiment that aims to teach the concepts of resultants torque and forces concerning the physical conditions for the equilibrium of a body. A plastic ruler is pivoted in its left extremity and supports a weight at the other extremity. The ruler is kept horizontal by a third force applied through a dynamometer. The calculation of the intensity of the force in terms of the weight supported by the system and the dynamometer positions on the ruler agrees with the experimental results.
\end{abstract}

Keywords: Equilibrium conditions of a rigid body. Moment of a force. Physics teaching.

\title{
I. INTRODUÇ̃̃O
}

Uma força pode ser capaz de transladar e/ou girar um corpo. Essas propriedades geralmente são estudadas separadamente, utilizando as leis da mecânica. Para que um corpo, inicialmente em repouso, não translade, a força resultante sobre o mesmo deve ser nula. Para que um corpo, inicialmente sem rotação, não gire, não deve existir torque resultante sobre o mesmo.

As duas condições previamente descritas devem ser obedecidas para que um corpo esteja em equilíbrio estático. Essa teoria é extensamente aplicada em Engenharia para determinação das configurações de estruturas como, por exemplo, estruturas de madeira e concreto, vigas, pontes, elementos de máquinas, etc. Além disso, o tema também é importante para compreender a mecânica envolvida nos músculos, tendões, ossos e articulações de humanos e outros animais.

\section{EQUAÇÕES PARA O EQUILÍBRIO MECÂNICO DO SISTEMA}

Considerando que a somatória vetorial das forças que atuam sobre a barra deve ser nula, o diagrama de corpo livre da mesma, mostrado na parte inferior da Figura 1, mostra que a equação pode para as intensidades das forças que atuam na barra em equilíbrio é

$$
R+T=P_{b}+P_{C},
$$

onde $R$ é a força na barra devido ao pivô, $T$ é a tração da corda vertical que sustenta o equilíbrio da barra através do dinamômetro, $P_{C}$ é o peso da carga e $P_{b}$, o peso da barra, que é representado no centro de massa da mesma.

A segunda condição necessária para garantir o equilíbrio estático do sistema é que o torque resultante seja nulo. Essa condição impede que rotações sejam iniciadas. O torque ou momento de uma força deve ser calculado com relação a um ponto arbitrário. No entanto, escolhas inteligentes para esse ponto devem ser feitas visando à solução de um problema 


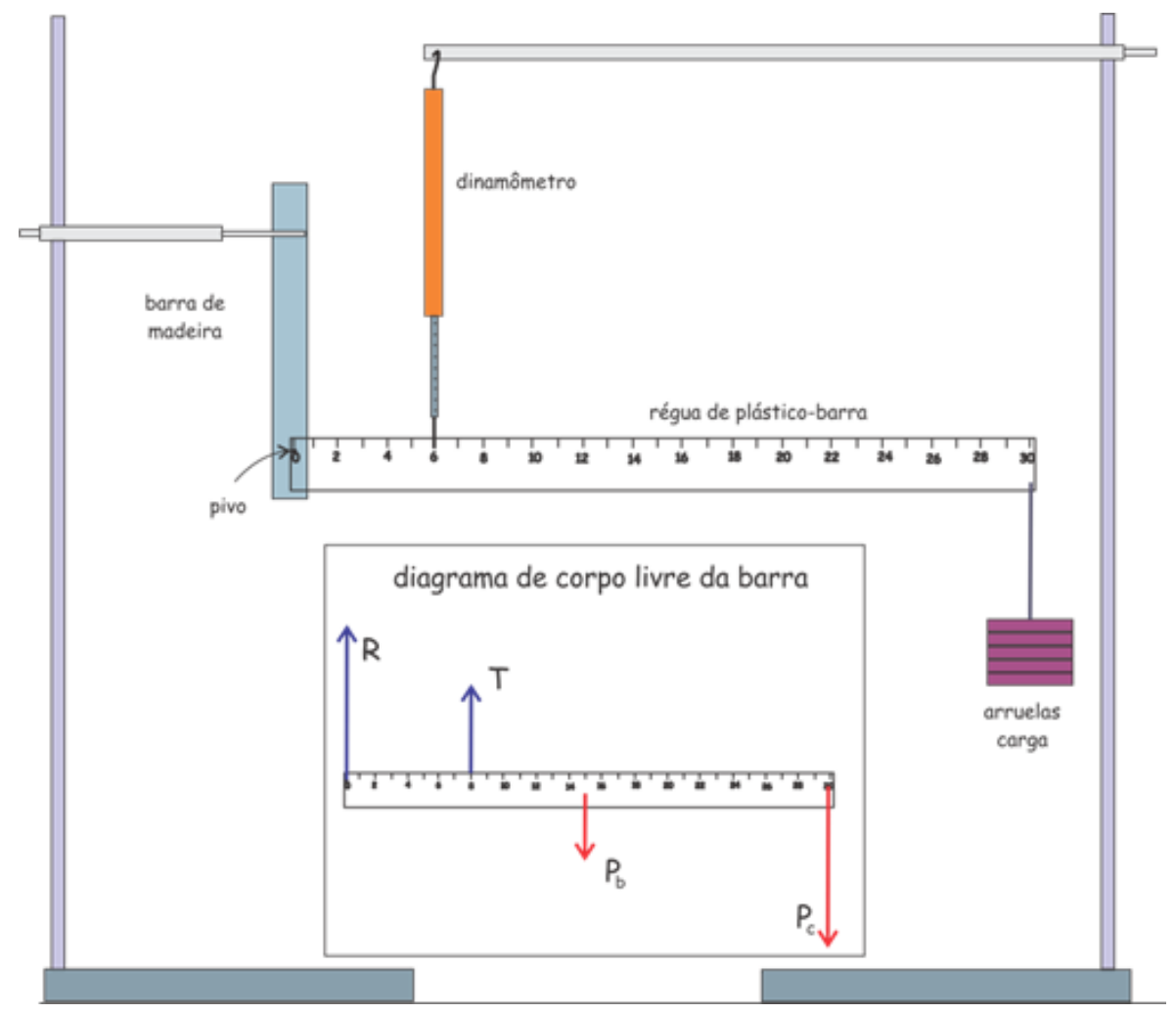

Figura 1: Ilustração do aparato experimental. Todas as forças envolvidas nesse artigo são perpendiculares ao comprimento da barra.

envolvendo equilíbrio. A equação matemática que mostra o torque de uma determinada força com relação a um determinado ponto arbitrário é

$$
\vec{\tau}=\vec{r} \times \vec{F},
$$

onde $\vec{r}$ é o vetor posição que se origina no ponto de referência escolhido e tem fim no ponto de aplicação da força no corpo.

No caso do problema aqui proposto, como as forças são todas perpendiculares à barra, escolhendo-se o ponto de pivotamento como referência, o torque é dado simplesmente pelo produto da distância deste ponto até o ponto de aplicação da força, multiplicado pela intensidade da força.

Assim, o torque no sentido anti-horário, $\tau_{+}$, vale:

$$
\tau_{+}=T . d,
$$

onde $d$ é a distância do ponto de pivotamento até o ponto onde a força $T$ é aplicada através do dinamômetro.

Analogamente, o torque que tende a girar a barra no sentido horário, $\tau_{-}$, é dado por:

$$
\tau_{-}=-P_{b} \frac{c}{2}-P_{C} \cdot c
$$

onde $c$ é a distância do pivô até o ponto onde a carga atua na barra. Assim, para que ocorra 
o equilíbrio, esses torques devem ter mesma intensidade:

$$
T . d=P_{b} \frac{c}{2}+P_{C} c .
$$

\section{II.1. Força necessária $F$ para o equilíbrio em função da carga fixada na posição $c$}

Para analisar como varia a força que deve ser aplicada pelo dinamômetro em função da carga, isola-se $T$ na Eq.(5)

$$
T\left(P_{C}\right)=\frac{c}{d} P_{C}+P_{b} \frac{c}{2 d}
$$

Nessa equação, $d$ e $c$, bem como $P_{b}$ são constantes. O peso da carga $P_{c}$ é a variável independente, enquanto que $T$ ou $T\left(P_{C}\right)$ é a variável dependente. Assim, a Eq.(6) evidencia que a força $T$ exercida na barra pelo dinamômetro deve crescer linearmente em função do peso da carga.

\section{II.2. Tração $T$ em função da posição variável desta na barra}

Nesta seção, o objetivo é estudar como varia a força de tração $T$, exercida pelo dinamômetro, em função da posição deste na barra. Considerando os parâmetros peso da carga, $P_{C}$, peso da barra, $P_{b}$, e as distâncias $d$ e $c$ constantes, a Eq. (5) pode ser reescrita como

$$
T(d)=\frac{P_{b} \frac{c}{2}+P_{C} \cdot c}{d} .
$$

Nota-se que a força de tração, $T$ ou $T(d)$, é uma função de proporcionalidade inversa, representada graficamente por uma hipérbole. Quanto mais próximo o ponto de aplicação da força $T$ do ponto de pivotamento, maior deverá ser sua magnitude para manter o equilíbrio do sistema.

\section{EXPERIMENTO}

Antes de iniciar a descrição dos experimentos, cabe dizer como foram feitos os gráficos. Devido à necessidade de qualidade gráfica, os gráficos apresentados nesse artigo foram construídos utilizando-se o programa Origin ${ }^{\circledR}$, (Origin, 2003). No entanto, outros programas, como o Graph, (Graph, 2007), podem ser utilizados para esse fim. O Graph permite, além de outras facilidades, que sejam impressos pontos experimentais e funções matemáticas em um mesmo gráfico. Tal capacidade é fundamental para comparações entre modelos teóricos e dados experimentais. Além disso, o Graph é software de código livre e pode ser obtido no site indicado na referência.

A montagem experimental é a mesma descrita na Figura 1. Como cargas, foram utilizadas arruelas de aproximadamente $6 \mathrm{~g}$ cada. As arruelas foram presas à barra através de um arame. A régua utilizada é feita de material plástico e tem massa de $23 \mathrm{~g}$ e $30 \mathrm{~cm}$ de comprimento. $\mathrm{O}$ dinamômetro utilizado é capaz de medir até 2,1 N, com precisão de 0,1 N. 


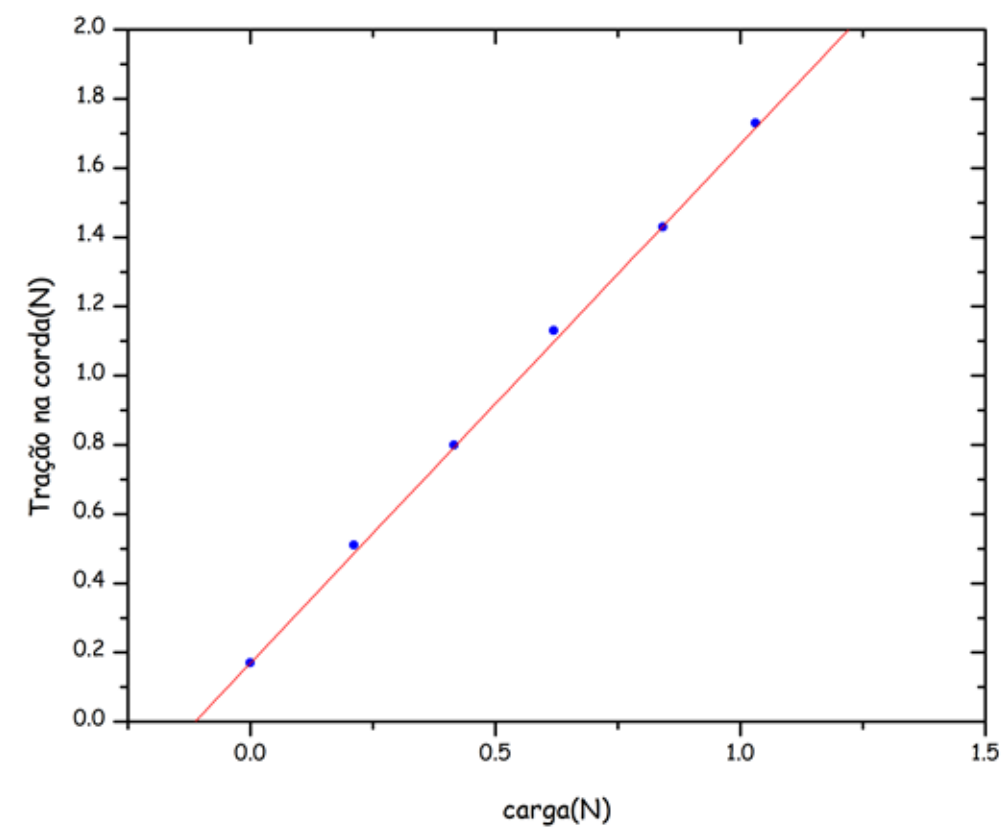

Figura 2: O gráfico evidencia a concordância quantitativa entre o modelo teórico, expresso pela linha contínua, com o experimento, círculos azuis.

\section{III.1. Parte I}

O objetivo nessa seção é testar a Eq.(6). Para isso, escolheu-se uma posição fixa, $d=20 \mathrm{~cm}$ para o dinamômetro e variou-se a quantidade de arruelas colocadas a distância fixa, $\mathrm{c}=30 \mathrm{~cm}$, do pivô. Para cada arruela adicionada, antes de realizar a leitura da força no dinamômetro, deve-se regular o sistema de modo que a barra permaneça na posição horizontal. Para melhor precisão, o peso do arame deve ser considerado.

Os pontos circulares no gráfico da Figura 2 representam os valores das forças de tração $T$ medidas com o dinamômetro para diferentes pesos da carga, $P_{C}$. A reta descreve a previsão teórica expressa por

$$
T\left(P_{C}\right)=1,5 P_{C}+0,169,
$$

que foi obtida substituindo os valores medidos na Eq.(6),

Pode-se observar que as medidas concordam qualitativamente e quantitativamente com o modelo, Eq.(8).

\section{III.2. Parte II}

O objetivo aqui é verificar na prática a previsão expressa pela Eq.(7), que mostra como deve variar a força $T$, medida pelo dinamômetro, em função da posição deste, $d$, ao suportar uma carga fixa $P_{C}$. Para isso, foram feitos pequenos furos na régua a cada $2 \mathrm{~cm}$ a partir do ponto $c$, onde as cargas são presas. Uma carga fixa de $43 \mathrm{~g}$ de arruelas foi fixada através de arame no ponto $c$ que dista $30 \mathrm{~cm}$ do pivô. Novamente, para realizar cada leitura no dinamômetro, deve-se ajustar a barra para posição horizontal. Os resultados obtidos são mostrados através dos círculos azuis no gráfico da Figura 3. 


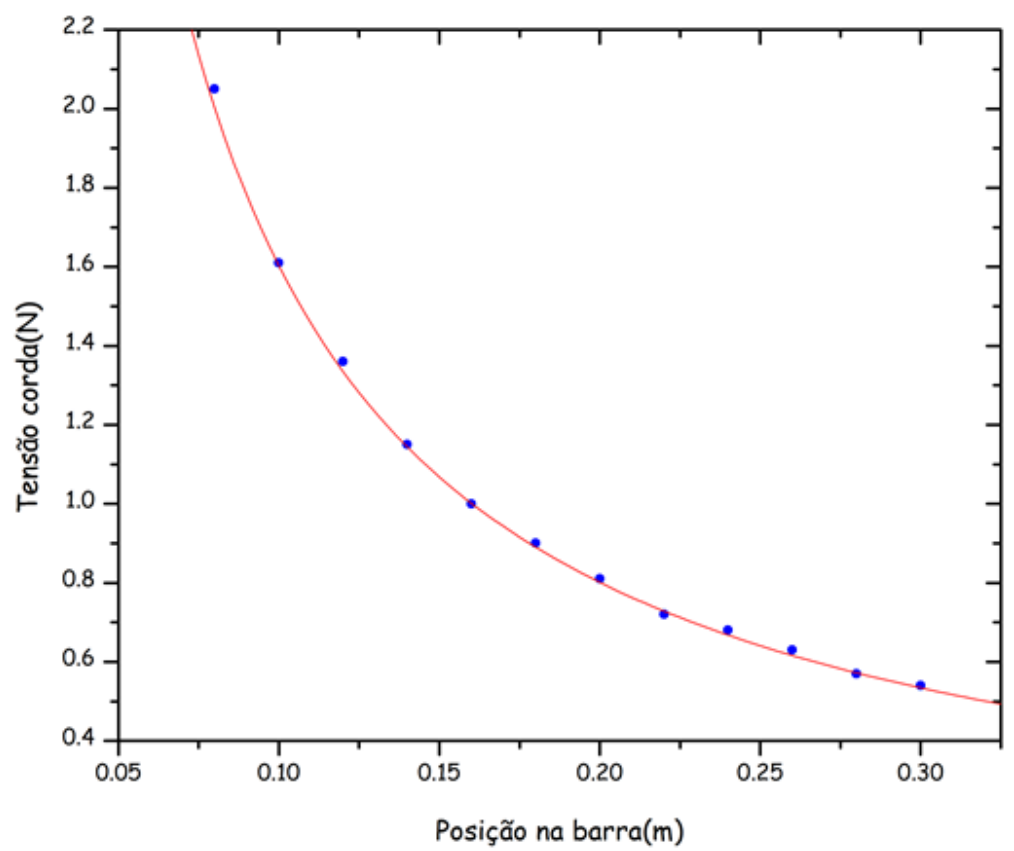

Figura 3: A função recíproca teórica $F(x)=0,16023 / d$, representada pela curva contínua, concorda com os dados obtidos no experimento.

A curva contínua vermelha do gráfico da Figura 3 representa a função

$$
T(d)=\frac{0,16023}{d}
$$

que foi obtida da Eq.(7) pela substituição dos valores dos parâmetros constantes $43 \mathrm{~g}$ da carga e $30 \mathrm{~cm}$ para a posição desta com relação ao ponto de pivotamento.

O gráfico mostra a boa concordância qualitativa e quantitativa entre as previsões do modelo teórico e as medidas experimentais, demonstrando a eficiência da teoria.

\section{CONClusões}

Esse artigo propõe um experimento simples e de baixo custo para estudar a teoria e a prática o equilíbrio de uma barra, em posição horizontal e equilibrada por três forças paralelas entre si e perpendiculares à barra. Foram deduzidas equações para duas situações de aplicação das forças na barra e foram executadas medidas experimentais visando verificar as previsões. Os gráficos construídos revelaram excelente concordância qualitativa e quantitativa entre as previsões do modelo teórico e as medidas experimentais. É consenso entre os autores que a presente proposta é adequada aos estudantes do nível médio e também estudantes universitários do primeiro ano de ciências e engenharia.

Diferentes competências dos estudantes podem ser trabalhadas ao realizar esse experimento, dentre elas pode-se citar: habilidades motoras para montagem do aparato e realização de medidas, prática de transformações de unidades físicas, construção e interpretação de gráficos, representação gráfica de funções matemáticas e tratamento teórico de um problema real. 


\section{REFERÊNCIAS}

ALONSO, M., FINN, E.J. Um curso Universitário-Mecânica. 2. ed., vol. 1, São Paulo, Bucher, 2014.

Graph [programa de computador- Versão 4.4.2, 2012)] Disponível livremente em: https:/ / www.padowan.dk/download/ Acesso em junho de 2019.

Origin [programa de computador- Versão 8.5, 2010] OriginLab Corp., Northampton, MA, 2003.

SEARS, F., ZEMANSKY, M., Física. Vol.1, 12 ed., São Paulo, Pearson Addison Wesley, 2008. 